\title{
Osteoarthritis Prevention and Treatment with a Plant-Based Diet
}

\author{
Stewart Rose* and Amanda Strombom \\ Plant-based Diets in Medicine, USA
}

Submission: November 14, 2019; Published: December 03, 2019

*Corresponding author: Stewart Rose, Plant-based Diets in Medicine, 12819 SE 38th St \#427, Bellevue, WA 98006, USA

\begin{abstract}
The prevalence of osteoarthritis is increasing not only because of longer life expectancy but also because of the modern lifestyle, in particular physical inactivity and diets low in plant foods and rich in saturated fats, which promote chronic low-grade inflammation and obesity. No proven disease-modifying or structure-modifying drugs for osteoarthritis are currently known. Consequently, pharmacologic treatment is directed at symptom relief. However, a whole food plant-based diet (WFPBD) has been shown to reduce the symptoms of osteoarthritis by reducing risk factors such as obesity, metabolic syndrome and Type II Diabetes. In an interventional study, a whole food plant-based diet was associated with a significant reduction in pain compared to an ordinary omnivorous diet, with statistically significant pain reduction seen as early as two weeks after initiation of dietary modification. Within the plant-based diet, several specific phytonutrients have been shown to reduce the symptoms and severity of osteoarthritis and can be considered active ingredients. Treating patients with a plant-based diet has the advantage of having no contraindications or adverse reactions and can be combined with any standard treatment. It also has the advantage of treating common comorbidities such as type 2 diabetes and coronary artery disease.
\end{abstract}

Keywords: BMI; Diabetes; Inflammation; Metabolic syndrome; Phytonutrients; Plant-based diet; Obesity; Osteoarthritis

Abbrevations: BMI: Body Mass Index; NRF2: Nuclear Factor Erythroid 2-related factor 2; OA Osteoarthritis; PQQ: Pyrroloquinoline Quinone; SFN: Sulforaphane; SIRT6: Sirtuin 6 T2DM Type 2 Diabetes Mellitus; WFPBD: Whole Food Plant Based Diet

\section{Introduction}

The prevalence of osteoarthritis is increasing not only because of longer life expectancy but also because of the modern lifestyle, in particular physical inactivity and diets low in plant foods and rich in saturated fats, which promote chronic lowgrade inflammation and obesity [1]. Osteoarthritis is believed to be a very complex multifactorial disease. It's a degenerative disease characterized by low grade inflammation in cartilage and synovium, resulting in the loss of joint structure and progressive deterioration of cartilage. Inflammation is one of the major drivers of the progression of osteoarthritis. Although the disease can be dependent on genetic and epigenetic factors, sex, ethnicity, lubricin level, apoptosis and age, it is also associated with obesity and being overweight, dietary factors, sedentary lifestyle and sport injuries [2].

\section{Epidemiology}

Vegetarians have a lower risk of osteoarthritis. One study showed that even light meat consumption once a week increased the risk of osteoarthritis by $31 \%$ in women and $19 \%$ in men, compared to vegetarians [3]. Obesity is the greatest modifiable risk factor for osteoarthritis (OA) [4-6]. Coggon et. al. [7] reported that subjects with a BMI greater than $30 \mathrm{~kg} / \mathrm{m} 2$ were 6.8 times more likely to develop knee $\mathrm{OA}$ than normal weight control subjects [7]. A recent meta-analysis reported that the pooled odds ratio for developing OA was 2.63 for obese subjects compared to normal weight control subjects [8]. Vegetarians and vegans have significantly lower BMIs on average. A study of American vegetarians and vegans found that that vegetarians had a mean BMI of 25.7 and vegans a mean BMI of 23.6 [9]. A European study found the average BMI of vegetarians and vegans to be 23.3 and 22.4 respectively for men and 22.8 and 21.8 for women [10]. A study of German vegans found an average BMI of 22.3 [11]. A study of vegetarian children found that they too had lower BMI's than their meat-eating counterparts with an average BMI of 17.3 in ages 6 to 11 and average of 20.0 ages 12-18 [12]. One study found the risk of being overweight or obese is $65 \%$ less for vegans and $46 \%$ less for vegetarians [13]. High body mass index (BMI) is a well-established risk factor for Type 2 Diabetes Mellitus (T2DM). T2DM increases the risk of development of severe osteoarthritis, necessitating arthroplasty 
independent of age and BMI. Diabetics face about double the risk of severe osteoarthritis suggesting that there is a strong metabolic component in the pathogenesis of osteoarthritis [14].

Metabolic factors are important for the development of osteoarthritis, as suggested by the following lines of evidence:

i. The association between obesity and OA extends beyond weight-bearing joints, suggesting that this link is not solely based on mechanical factors [15].

ii. Metabolic syndrome is significantly more common among subjects with than without OA $[16,17]$.

Two lines of experimental evidence may help to explain the link between type 2 diabetes and $\mathrm{OA}$ :

a. chondrocytes express the GLUT/SLC2A, and high blood glucose levels shift the synthesis pattern of chondrocytes from type II collagen to reactive oxygen species, potentially mediating cartilage destruction [18-20].

b. Advanced glycosylation end products, elicited by sustained hyperglycemia, can stimulate chondrocyte expression of proinflammatory and prodegenerative proteins via the receptor for advanced glycosylation end products [21-23].

Vegetarians have a $56 \%$ reduced risk of metabolic syndrome [24]. Vegetarian and vegans also have a substantially lower risk of Type 2 Diabetes. The consumption of meat and the increase in risk of T2DM in a dose dependent manner has been established since at least 1985 [25]. More recently, a large, well-regarded study showed that semi-vegetarians reduced their risk of T2DM by $38 \%$, pesco vegetarians by $51 \%$, vegetarians by $61 \%$ and vegans by $78 \%$. This indicates a dose-response relationship between risk reduction and amount of plant foods in the diet [26]. Therefore, it follows that those following a plant-based diet would have a lower risk of osteoarthritis, because of their lower BMI and lower risk of type 2 diabetes.

\section{Intervention}

Individuals diagnosed with arthritis can take steps to improve their diet quality as a possible route to reduce arthritis symptoms and maintain a healthy body weight [27]. In an interventional study, a whole food plant-based diet was associated with a significant reduction in pain compared to an ordinary omnivorous diet, with statistically significant pain reduction seen as early as two weeks after initiation of dietary modification [28]. The primary mechanism by which diet reduces subjective pain may be as a result of normalization of the fatty acid profile and reduction in exposure to inflammatory protein precursors [28].

The plant-based dietary profile (low fat, high fiber) can lead to a diet that is less energy dense and so results in a significant reduction in caloric intake. Despite reductions in calorie intake, the WFPB diet is associated with increased nutrient density as well as increased concentrations of several vitamins and trace minerals. Therefore, the WFPB diet group may have taken in fewer calories than the treated group while encouraged to eat to satiety without calorie counting. The reduction in mean body weight was achieved with no attempt to limit calorie intake in this study [28]. While not a fully plant-based diet, the Mediterranean Diet is high in plant foods and low in meat. In one study the Mediterranean Diet was associated with better quality of life and decreased pain and disability in patients with osteoarthritis [29].

\section{Specific Phytonutrients and their Mechanisms}

Several phytonutrients have been shown to reduce the symptoms of osteoarthritis. Dietary phytonutrients (phytochemicals) are found in plant-based foods such as fruits, vegetables and grains, and may be categorized in a nested hierarchical manner with many hundred individual phytochemicals identified to date [30]. Phytonutrients are "bioactive compounds" that have the ability to interact with one or more compounds in living tissue, resulting in an effect on human health [31]. Exploring dietary phytochemical intake from foods may complement current dietary strategies for the management of osteoarthritis [32].

Cyanidin is the major component of anthocyanins commonly found in plant foods. Increasing evidence has shown that cyanidin exhibits anti-inflammatory effects in a variety of diseases. Cyanidin is a rare Sirtuin 6 (SIRT6 or Sirt6) activator. SIRT6 is a stress responsive protein deacetylase and mono-ADP ribosyltransferase enzyme encoded by the SIRT6 gene [33]. SIRT6 functions in multiple molecular pathways related to aging, including DNA repair, telomere maintenance, glycolysis and inflammation [33]. Cyanidin could inhibit the NF- $\mathrm{KB}$ pathway in IL-1 $\beta$-stimulated human osteoarthritic chondrocytes and its effect may to some extent depend on SIRT6 activation, suggesting that cyanidin may exert a protective effect through regulating the Sirt6/NF- $\kappa$ B signaling axis. Moreover, an in vivo study also showed that cyanidin ameliorated the development of osteoarthritis in surgical destabilization of the medial meniscus in mouse osteoarthritic models [34]. Sulforaphane (SFN), a phytonutrient found in broccoli, has been reported to regulate signaling pathways relevant to chronic diseases. SFN inhibits the expression of key metalloproteinases implicated in osteoarthritis, independently of $\mathrm{Nrf} 2$, and blocks inflammation at the level of NF- $\mathrm{kB}$ to protect against cartilage destruction in vitro and in vivo [35].

Pyrroloquinoline quinone (PQQ) is a compound found ubiquitously in plants, many simple and single cell eukaryotes (e.g., yeast), and certain bacteria [36]. Certain foods such as green peppers, cabbage, spinach, papaya and kiwi fruit have higher levels of PQQ [37]. Accumulating evidence suggests that oxidative stress plays an important role in the progression of 


\section{Orthopedics and Rheumatology Open Access Journal (OROAJ)}

osteoarthritis, and PQQ is considered a strong antioxidant [38]. Supplementation largely prevented these alterations in mice. Articular surfaces were maintained, while the thickness of articular cartilage and the abundance of cartilage matrix protein were also positively affected [38].

Supplementation with vitamin D over four years was associated with significantly less progression of knee joint abnormalities. Given the observational nature of this study, future longitudinal randomized controlled trials of vitamin D supplementation are warranted [39]. Dietary polyphenols have been studied for their anti-inflammatory properties and potential anabolic effects on the cartilage cells. Blueberries are widely consumed and are high in dietary polyphenols, therefore regular consumption of blueberries may help improve osteoarthritis [40]. The findings of one study shows that daily incorporation of whole blueberries may reduce pain, stiffness, and difficulty to perform daily activities, while improving gait performance, and would therefore improve quality of life in individuals with symptomatic knee osteoarthritis. (40). A study showed that strawberries may have significant analgesic and anti-inflammatory effects in obese adults with established knee osteoarthritis [41]. Another study showed that strawberries lowered TNF- $\alpha$, and lipid peroxidation products in obese adults with knee osteoarthritis [42].

\section{Discussion}

Osteoarthritis is a chronic disease. While several medications have shown efficacy in reducing the symptoms, their daily use in the long term can be problematic. However, a plant-based diet can help treat the symptoms without long term problems, and with long term benefits for osteoarthritic symptoms and common comorbidities such coronary artery disease. There are no contraindications or adverse reactions to the treatment of osteoarthritis with a plant-based diet. A vegetarian diet lowers the risk of osteoarthritis. "As well as treating those who already have the condition, you need to be able to tell healthy people how to protect their joints into the future," study author Dr. Ian Clark, at the University of East Anglia in Norwich, U.K, said in a statement. „There is currently no way to prevent the disease pharmaceutically and you cannot give healthy people drugs unnecessarily, so this is where diet could be a safe alternative." For those already with osteoarthritis a whole food plant-based diet reduces the symptoms of osteoarthritis. Even diets that were mostly plant-based showed some effect on symptoms. While there are still a large number to investigate, several phytonutrients and plant foods have already shown efficacy in reducing the symptoms of osteoarthritis. Understanding the effect of dietary phytochemical intake from foods on osteoarthritis and its long-term outcomes may inform public health strategies for osteoarthritis prevention and management, reducing healthcare costs globally.

\section{Conflict of Interest}

The authors state that they have no conflict of interest.

\section{References}

1. Biver E, Berenbaum F, Valdes A, Araujo de Carvalho I, Bindels L, et al. (2019) Gut microbiota and osteoarthritis management: An expert consensus of the European society for clinical and economic aspects of osteoporosis, osteoarthritis and musculoskeletal diseases (ESCEO). Ageing Res Rev 55: 100946

2. Musumeci G, Aiello F, Szychlinska M, DiRosa M, Castrogiovanni P, et al (2015) Osteoarthritis in the XXIst century: risk factors and behaviours that influence disease onset and progression. Int J Mol Sci 16(3): 6093 6112.

3. Hailu A, Knutsen , Fraser GE (2006) Associations between meat consumption and the prevalence of degenerative arthritis and soft tissue disorders in the adventist health study, California U.S.A. J Nutr Health Aging 10(1): 7-14

4. Anandacoomarasamy A, Caterson I, Sambrook P, Fransen M, March L (2008) The impact of obesity on the musculoskeletal system. Int J Obes (Lond) 32(2): 211-222.

5. Spector TD, Hart D, Doyle DV (1994) Incidence and progression of osteoarthritis in women with unilateral knee disease in the general population: the effect of obesity. Ann Rheum Dis 53(9): 565-568.

6. Szoeke C, Dennerstein L, Guthrie J, Clark M, Cicuttini F (2006) The relationship between prospectively assessed body weight and physical activity and prevalence of radiological knee osteoarthritis in postmenopausal women. J Rheumatol 33(9): 1835-1840.

7. Coggon D, Reading I, Croft P, McLaren M, Barrett D, et al. (2001) Knee osteoarthritis and obesity. Int J Obes Relat Metab Disord 25(5): 622627.

8. Blagojevic M, Jinks C, Jeffery A, Jordan K (2010) Risk factors for onset of osteoarthritis of the knee in older adults: a systematic review and meta-analysis. Osteoarthritis Cartilage 18(1): 24-33

9. Tonstad S, Butler T, Yan R, Fraser G (2009) Type of Vegetarian Diet Body Weight, and Prevalence of Type 2 Diabetes. Diabetes Care 32(5): 791-796.

10. Bradbury K, Crowe F, Appleby P, Schmidt J, RC T, et al.(2014) Serum concentrations of cholesterol, apolipoprotein A-I, and apolipoprotein $B$ in a total of 1694 meat-eaters, fish-eaters, vegetarians, and vegans. Eur J Clin Nutr 68(2): 178-183.

11. Waldmann A, Koschizke J, Leitzmann C, Hahn A (2005) German vegan study: diet, life-style factors, and cardiovascular risk profile. Ann Nutr Metab 49(6): 366-372.

12. Haddad E, Tanzman J (2003) What do vegetarians in the United States eat? Am J Clin Nutr 78(3): 626S-632S.

13. Newby P, Tucker K, Wolk A (2005) Risk of overweight and obesity among semivegetarian, lactovegetarian, and vegan women. Am J Clin Nutr 81(6): 1267-1274.

14. Schett G, Kleyer A, Perricone C, Sahinbegovic E, Iagnocco A, et al (2013) Diabetes is an independent predictor for severe osteoarthritis: results from a longitudinal cohort study. Diabetes Care 36(2): 403-409.

15. Pottie P, Presle N, Terlain B, Netter P, Mainard D, et al. (2006) Obesity and osteoarthritis: more complex than predicted! Ann Rheum Dis. 65(11): 1403-1405.

16. Puenpatom R, Victor TW (2009) Increased prevalence of metabolic syndrome in individuals with osteoarthritis: an analysis of NHANES III data. Postgrad Med 121(6): 9-20. 
17. Cimmino M, Cutolo M (1990) Plasma glucose concentration in symptomatic osteoarthritis: a clinical and epidemiological survey. Clin Exp Rheumatol 8(3): 251-257.

18. Shikhman A, Brinson D, Valbracht J, Lotz M (2001) Cytokine regulation of facilitated glucose transport in human articular chondrocytes. J Immunol 167(12): 7001-7008.

19. Rosa S, Gonçalves J, Judas F, Mobasheri A, Lopes C, et al. (2009) Impaired glucose transporter-1 degradation and increased glucose transport and oxidative stress in response to high glucose in chondrocytes from osteoarthritic versus normal human cartilage. Arthritis Res Ther 11(3): R80.

20. Henrotin Y, Bruckner P, Pujol J (2003) The role of reactive oxygen species in homeostasis and degradation of cartilage. Osteoarthritis Cartilage 11(10): 747-755.

21. Steenvoorden M, Huizinga T, Verzijl N, Bank R, Ronday H, et al. (2006) Activation of receptor for advanced glycation end products in osteoarthritis leads to increased stimulation of chondrocytes and synoviocytes. Arthritis Rheum 54(1): 253-263.

22. Loeser R, Yammani R, Carlson C, Chen H, Cole A, et al. (2005) Articular chondrocytes express the receptor for advanced glycation end products: Potential role in osteoarthritis. Arthritis Rheum 52(8): 23762385.

23. Verzijl N, DeGroot J, Zaken CB, Brau-Benjamin O, Maroudas A, et al (2002) Crosslinking by advanced glycation end products increases the stiffness of the collagen network in human articular cartilage: A possible mechanism through which age is a risk factor for osteoarthritis. Arthritis Rheum. 46(1): 114-123.

24. Rizzo N, Sabaté J, Jaceldo-Siegl K, Fraser G (2011) Vegetarian dietary patterns are associated with a lower risk of metabolic syndrome: the adventist health study 2. Diabetes Care 34(5): 1225-1257.

25. Snowdon D, Phillips RL (1985) Does a vegetarian diet reduce the occurrence of diabetes? Am J Public Health 75(5): 507-512.

26. Fraser GE (2009) Vegetarian diets: what do we know of their effects on common chronic diseases? Am J Clin Nutr 89(5): 1607S-1612S.

27. Comee L, Taylor C, Nahikian-Nelms M, Ganesan L, Krok-Schoen J (2019) Dietary patterns and nutrient intake of individuals with rheumatoid arthritis and osteoarthritis in the United States. Nutrition 6768: 110533.

28. Clinton C, O'Brien S, Law J, Renier C, Wendt M (2015) Whole-Foods, Plant-Based Diet Alleviates the Symptoms of Osteoarthritis. Arthritis 2015: 708152.

29. Veronese N, Stubbs B, Noale M, Solmi M, Luchini C, et al. (2016) Adherence to the Mediterranean diet is associated with better quality of life: data from the Osteoarthritis Initiative. Am J Clin Nutr 104(5): 1403-1409.
30. Probst Y, Guan V, Kent K (2017) Dietary phytochemical intake from foods and health outcomes: a systematic review protocol and preliminary scoping. BMJ Open 7(2): e013337.

31. Biesalski H, Dragsted L, Elmadfa I, Grossklaus R, Müller M, et al. (2009) Bioactive compounds: definition and assessment of activity. Nutrition 25(11-12): 1202-1205.

32. Guan V, Mobasheric A, Probst YC (2019) A systematic review of osteoarthritis prevention and management with dietary phytochemicals from foods. Maturitas 122: 35-43.

33. Frye RA (2000) Phylogenetic classification of prokaryotic and eukaryotic Sir2-like proteins. Biochem Biophys Res Commun 273(2): 793798.

34. Jiang C, Sun Z, Hu J, Jin Y, Guo Q et al. (2019) Cyanidin ameliorates the progression of osteoarthritis via the Sirt6/NF- $\mathrm{B}$ axis in vitro and in vivo. Food Funct 10(9): 5873-5885.

35. Davidson R, Jupp O, deFerrars R, Kay C, Culley K, et al. (2013) Sulforaphane Represses Matrix-Degrading proteases and protects cartilage from destruction in vitro and in vivo. Arthritis Rheum 65(12): 31303140 .

36. Jonscher K, Rucker R (2019) Pyrroloquinoline Quinone Its Profile, Effects on the Liver and Implications for Health and Disease Prevention. In: Watson R, Preedy V, eds. Dietary Interventions in Liver Disease: Foods, Nutrients, and Dietary Supplements: Academic Press, USA.

37. Kumazawa T, Sato K, Seno H, Ishii A, Suzuki O (1995) Levels of pyrroloquinoline quinone in various foods. Biochem J 307(Pt 2): 331-333.

38. Qin R, Sun J, Wu J, Chen L (2019) Pyrroloquinoline quinone prevents knee osteoarthritis by inhibiting oxidative stress and chondrocyte senescence. Am J Transl Res 11(3): 1460-1472.

39. Joseph G, McCulloch C, Nevitt M, Neumann J, Lynch J, et al. (2019) Associations between Vitamin $\mathrm{C}$ and D Intake and Cartilage Composition and Knee Joint Morphology over 4 years: Data from the Osteoarthritis Initiative. Arthritis Care Res (Hoboken).

40. Du C, Smith A, Avalos M, South S, Crabtree K, et al. (2019) Blueberries Improve Pain, Gait Performance, and Inflammation in Individuals with Symptomatic Knee Osteoarthritis. Nutrients 11(2): E290.

41. Schell J, Scofield R, Barrett J, Kurien B, Betts N, et al. (2017) Strawberries Improve Pain and Inflammation in Obese Adults with Radiographic Evidence of Knee Osteoarthritis. Nutrients 9(9): E949.

42. Basu A, Kurien B, Tran H, Byrd B, Maher J, et al. (2018) Strawberries decrease circulating levels of tumor necrosis factor and lipid peroxides in obese adults with knee osteoarthritis. Food Funct 9(12): 6218-6226. 


\section{Your next submission with Juniper Publishers} will reach you the below assets

- Quality Editorial service

- Swift Peer Review

- Reprints availability

- E-prints Service

- Manuscript Podcast for convenient understanding

- Global attainment for your research

- Manuscript accessibility in different formats ( Pdf, E-pub, Full Text, Audio)

- Unceasing customer service

Track the below URL for one-step submission https://juniperpublishers.com/online-submission.php 